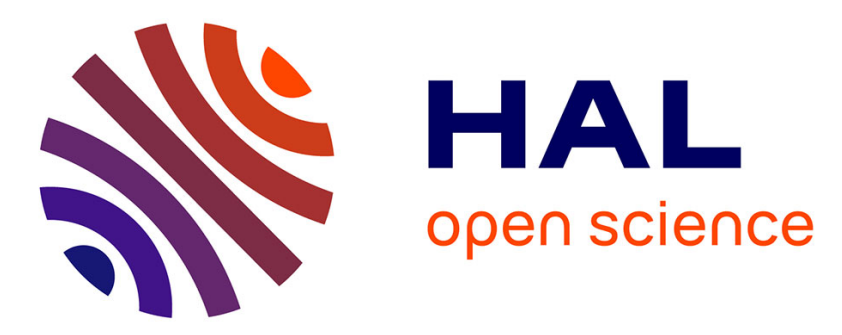

\title{
From Obstacle-Based Space Partitioning to Corridors and Path Planning. A Convex Lifting Approach
}

\author{
Daniel Ioan, Sorin Olaru, Ionela Prodan, Florin Stoican, Silviu-Iulian
} Niculescu

\section{- To cite this version:}

Daniel Ioan, Sorin Olaru, Ionela Prodan, Florin Stoican, Silviu-Iulian Niculescu. From Obstacle-Based Space Partitioning to Corridors and Path Planning. A Convex Lifting Approach. IEEE Control Systems Letters, 2020, 4 (1), pp.79-84. 10.1109/LCSYS.2019.2922414 . hal-02328848

\section{HAL Id: hal-02328848 \\ https://hal-centralesupelec.archives-ouvertes.fr/hal-02328848}

Submitted on 29 Jul 2020

HAL is a multi-disciplinary open access archive for the deposit and dissemination of scientific research documents, whether they are published or not. The documents may come from teaching and research institutions in France or abroad, or from public or private research centers.
L'archive ouverte pluridisciplinaire HAL, est destinée au dépôt et à la diffusion de documents scientifiques de niveau recherche, publiés ou non, émanant des établissements d'enseignement et de recherche français ou étrangers, des laboratoires publics ou privés. 


\title{
From Obstacle-based Space Partitioning to Corridors and Path Planning. A Convex Lifting Approach.
}

\author{
Daniel Ioan ${ }^{1}$, Sorin Olaru ${ }^{1}$, Ionela Prodan ${ }^{2}$, Florin Stoican $^{3}$, Silviu-Iulian Niculescu ${ }^{1}$
}

\begin{abstract}
This paper proposes a novel methodology for path generation in known and congested multi-obstacle environments. Our aim is to solve an open problem in navigation within such environments: the feasible space partitioning in accordance with the distribution of obstacles. It is shown that such a partitioning is a key concept towards the generation of a corridor in cluttered environments. Once a corridor between an initial and a final position is generated, the selection of a path is considerably simplified in comparison with the methods which explore the original non-convex feasible regions of the environment. The core of the methodology presented here is the construction of a convex lifting which boils down to a convex optimization. The paper covers both the mathematical foundations and the computational details of the implementation and aims to illustrate the concepts with geometrical examples.
\end{abstract}

\section{INTRODUCTION}

Navigation through multi-obstacle environments has received substantial attention in the control and robotics communities [1] due to its numerous applications, e.g., monitoring or surveillance [2]. From a mathematical point of view, the main difficulty comes from the non-convexity of the feasible regions in the motion space and consequently in the lack of connectivity in the solution space.

In the literature, motion planning is usually divided in three tasks. The first task is path planning which refers to the construction of a route in the navigation space without an explicit parametrization in time. Next, the trajectory planning represents the search of feasible trajectories which respect the generated path and the constraints given by the dynamical and physical limitations. Lastly, the low-level control consists in elaborating a reliable feedback control strategy such that the considered agent follows the obtained trajectory.

The existing approaches may be classified into two main categories. The former, optimization-based strategies, e.g. mixed-integer formulations [3], [4], potential field methods [5], [6], [7], or convexification techniques [8], merge the path and trajectory planning tasks at the expense of a higher computational complexity in the case of congested multiobstacle environments. The latter, sample-based methods [9], [10], relies on the construction of a graph and focuses with predilection on the first task of motion planning. Regardless of the method, an important aspect is the way in which the

\footnotetext{
${ }^{1}$ Laboratory of Signals and Systems, Univ. Paris-Sud-CentraleSupelecCNRS, Université Paris Saclay. \{daniel.ioan, sorin.olaru, silviu.niculescu\}el2s.centralesupelec.fr

${ }^{2}$ Univ. Grenoble Alpes, Grenoble INP^, LCIS, F-26000, Valence, France. ionela.prodanelcis.grenoble-inp.fr

* Institute of Engineering Univ. Grenoble Alpes.

${ }^{3}$ Department of Automatic Control and Systems Engineering, UPB, Romania florin.stoicandacse.pub.ro
}

environment is modeled, a popular practice being the use of convex sets, either polytopes [4] or ellipsoids [11] with different artifacts on the local or global feasibility and on the optimality [12].

In the present paper, the focus is on the path-planning level with a primary objective of global feasibility. The differential constraints are discarded from the problem formulation as well as the limitations that can appear in motion planning due to limited steering or energy. Optimality is a secondary objective after the generation of a geometric path which ensures the avoidance of obstacles and has the potential to explicitly describe a feasible corridor as in [13].

From the mathematical point of view the solution will exploit convex lifting [14], previously employed in constrained control and PWA (piecewise affine) control implementations. This notion proves to be particularly efficient for constructing partitions and will be used here to characterize the navigation space. This versatile optimization-based approach for the construction of a partition starting from the obstacles can be understood as a convexification procedure for the characterization of the non-convex feasible regions in the motion space.

The main contributions of this paper are the following: i) provide a partitioning of the navigation space based on convex lifting; ii) construct feasible corridors based on a graph of interconnections in the multi-obstacle environment; iii) propose a path with obstacle avoidance guarantees.

Moreover, our solution is not restricted to $\mathbb{R}^{2}$, nor $\mathbb{R}^{3}$ and provides a generic path-generation technique in any finite dimensional space with obstacles.

Notation: The Minkowski sum of two sets is denoted as $A \oplus$ $B=\{x: x=a+b, a \in A, b \in B\}$. Given a compact set $S \in \mathbb{R}^{n}, \mathcal{C}_{X}(S)$ denotes the complement of $S$ over $X \in$ $\mathbb{R}^{d}$, and $\operatorname{int}(S)$ its interior. $\operatorname{Conv}(S)$ is the convex hull of $S \in \mathbb{R}^{n}, \operatorname{Com}(S)$ the space of compact subsets of $S$, and $\mathcal{V}(S)$ the set of its extreme points (possibly infinite collection of points spanning $\operatorname{Conv}(S)$ ). For a polyhedron $P \in \mathbb{R}^{d}$, $\mathcal{V}(P)$ is the (finite) set of its vertices, and $\mathcal{F}_{i}^{k}(P)$ is the $i$-th face of the dimension $k<d$. Any polytope (i.e. a bounded polyhedron) has a dual representation in terms of intersection of half-spaces or convex hull of extreme points: $P=\{x$ : $\left.s_{i}^{\top} x \leq r_{i}, \forall i\right\}=\left\{x: x=\sum \alpha_{j} v_{j}, \sum \alpha_{j}=1, \alpha_{j} \geq 0, \forall j\right\}$. $\mathbb{B}_{p, r}=\left\{x \in \mathbb{R}^{d}:\|x-p\| \leq r\right\}$ is a ball of radius $r \geq 0$ centered in $p \in \mathbb{R}^{d}$ w.r.t. a given norm.

\section{Preliminaries and Problem Statement}

Consider a finite dimensional output space $\mathbb{R}^{d}$ and a finite number of non-overlapping regions $P_{j} \in$ 
$\operatorname{Com}\left(\mathbb{R}^{d}\right), j \in \mathcal{I}=\left\{1, \ldots, N_{o}\right\}$ describing obstacles:

$$
\mathbb{P}=\bigcup_{j=1}^{N_{o}} P_{j} ; P_{i} \cap P_{j}=\emptyset, \forall i \neq j .
$$

The union of obstacles (1) lies in a bounded ${ }^{1}$ cluttered environment $\mathbb{X}$ :

$$
\mathbb{P} \subset \operatorname{int}(\mathbb{X}) \subset \mathbb{R}^{d}
$$

while the obstacle-free/feasible domain is $\mathcal{C}_{\mathbb{X}}(\mathbb{P}) \triangleq \mathbb{X} \backslash \mathbb{P}$.

Definition 1: Given the obstacles $\mathbb{P}$, a corridor between two points $x_{0}, x_{f} \in \operatorname{int}\left(\mathcal{C}_{\mathbb{X}}(\mathbb{P})\right)$ is enabled by the existence of two continuous functions:

$$
\begin{aligned}
& \gamma:[0,1] \rightarrow \mathcal{C}_{\mathbb{X}}(\mathbb{P}) \\
& \rho:[0,1] \rightarrow \mathbb{R}_{>0}
\end{aligned}
$$

satisfying

$$
\begin{gathered}
\gamma(0)=x_{0}, \gamma(1)=x_{f} \\
\gamma(\theta) \oplus \mathbb{B}_{0, \rho(\theta)} \subset \mathcal{C}_{\mathbb{X}}(\mathbb{P}), \forall \theta \in[0,1]
\end{gathered}
$$

Based on (3)-(6) the corridor is defined as:

$$
\Pi=\left\{x \in \mathbb{R}^{d}: \exists \theta \in[0,1] \text { s.t. } x \in \gamma(\theta) \oplus \mathbb{B}_{0, \rho(\theta)}\right\} .
$$

The objective of the present work is the construction of corridors in a cluttered environment.

Remark 1: Corridor construction encompasses classical path planning. Indeed, the corridor can be understood as a compact family of feasible paths between the initial and final point. Obviously, the selection of a path within a corridor is conceptually simpler in comparison with the direct search of a path avoiding the obstacles (provided that a corridor has already been found). Additionally, given a path within a corridor, the distance to the boundary of the corridor represents a robustness margin for the trajectory.

The notion of distance in this context is related to the characterization of the nearest obstacles and indirectly leads to the partitioning of the cluttered environment according to the distribution of obstacles.

Definition 2: A family of sets $\left\{X_{i}\right\}_{i \in \mathcal{I}}$ verifying:

i) $\mathbb{X}=\bigcup_{i=1}^{N_{o}} X_{i}$

ii) $\operatorname{int}\left(X_{i}\right) \bigcap \operatorname{int}\left(X_{j}\right)=\emptyset, \forall i \neq j \in \mathcal{I}$,

iii) $P_{i} \subset \operatorname{int}\left(X_{i}\right), \forall i \in\left\{1 \ldots, N_{o}\right\}$

is called a partition of $\mathbb{X}$ induced by the obstacles $\mathbb{P}$.

Definition 3: If the sets $\mathbb{X}$ and $X_{i}, \forall i$ in Def. 2 are polyhedral, then $\mathbb{X}=\bigcup_{i=1}^{N_{o}} X_{i}$ is called a polyhedral partition. $\checkmark$

Problem formulation: The paper deals with three issues:

P1) Given convex obstacles $\mathbb{P}$, describe a partition of the cluttered environment around them. Provide a constructive algorithm for the case of the polytopic obstacles. (Section III)

P2) Given any two points in the cluttered environment $x_{0}, x_{f} \in \operatorname{int}\left(\mathcal{C}_{\mathbb{X}}(\mathbb{P})\right)$, construct a corridor or provide a certificate of infeasibility. (Section IV)

P3) Given a non-empty corridor, select according to some performance criteria a continuous path $\pi:[0,1] \rightarrow \Pi$

\footnotetext{
${ }^{1}$ Always possible, due to the boundedness assumptions on $P_{j}, j \in \mathcal{I}$
}

guaranteeing collision avoidance, i.e. $\pi(\theta) \cap \mathbb{P}=\emptyset, \forall \theta \in$ $[0,1]$. (Section III, Algorithm 1)

Since there are in the literature works which treat in detail problem P3) using à priori defined corridors, see, e.g., [15], we focus hereinafter on providing a methodical solution of first two problems P1) and P2), respectively.

Before the main development, let us briefly show that the problems formulated above are not trivial. An intuitive, but inadequate idea for partitioning of the cluttered environment is related to a Voronoi-like construction. Indeed, the points in the classical Voronoi partitioning can be replaced by the collection of obstacles. Well-known from convex analysis [16], the existence of the separating hyperplane is guaranteed for any pair of disjoint convex sets. Obviously, as $P_{i} \cap P_{j}=$ $\emptyset, \forall i \neq j$, these separating hyperplanes are candidates for the supporting hyperplanes of $X_{i} \supset P_{i}$. However, associating to each obstacle $P_{i}$ a cell : $\tilde{X}_{i}=\left\{x \in \mathbb{R}^{d}: H_{i} x \leq k_{i}\right\}$, where $H_{i}$ and $k_{i}$ are composed by the separating hyperplane between the obstacle $P_{i}$ and all of its neighbors, does not lead to a partition as defined in Def. 2 . The scenario depicted in Fig. 1, where the union of cells $\tilde{X}_{i}$ does not cover the entire space, is a simple example of this issue.

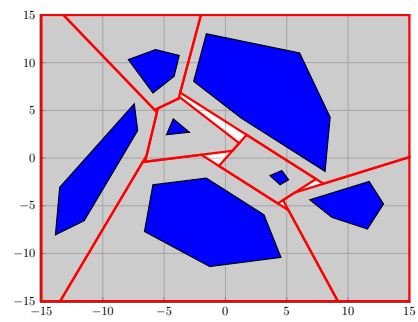

Fig. 1: Space-partitioning using separating hyperplanes.

Furthermore, space partitioning has an instrumental role w.r.t. motion planning methods and, specifically, those employing a path planner. The vast majority of these methods relies on constructing a graph as a result of the workspace partitioning, e.g., by using a grid of square/cubic cells [17] or Voronoi diagrams [18]. The construction of such diagrams relies on the selection of certain points in the workspace: either mass (or Chebyshev) centers of the obstacles or a set of points approximating the boundary of the obstacles [12]. Nevertheless, the resulting diagrams either do not satisfy the characteristics of a partition (as in Def. 2) or the resulting graph needs an additional processing step in order to remove redundant nodes and edges and whose overall complexity is not negligible ${ }^{2}$.

With respect to P1) and P2), to the best of the authors' knowledge, there does not exist any systematic method which certifies the feasibility of the global corridor/path planning and its constructive solution. However, as stated in Section I, there is a broad variety of techniques which aim to fulfill the goal by partitioning/dividing the global task into individually manageable components. Thus, recently, methods able to precompute feasible (geometric) paths, as in P3), have received

\footnotetext{
${ }^{2}$ Moreover, these solutions are generally defined only for the planar case.
} 
a considerable attention under various forms, e.g., waypoint trajectory planning [12].

The next sections show that an association $P_{i} \rightarrow X_{i}$ leading to a partition is achievable in a tractable manner through the extension of the notion of convex lifting [14] to the present obstacle avoidance setting.

\section{CONVEX LIFTING FOR SPACE PARTITIONING}

Definition 4: Given a collection of obstacles $\mathbb{P}=\bigcup_{j=1}^{N_{o}} P_{j}$ with $P_{i} \cap P_{j}=\emptyset, \forall i \neq j$, as defined in (1), and a partitioning of the cluttered environment $\mathbb{X} \supset \mathbb{P}$, as in Def. 2 , the function $z: \mathbb{X} \rightarrow \mathbb{R}$ is called a PWA lifting if:

$$
z(x)=a_{i}^{\top} x+b_{i}, x \in X_{i},
$$

with $X_{i}$ satisfying $\operatorname{int}\left(X_{i}\right) \supset P_{i}, \forall i, a_{i} \in \mathbb{R}^{d}$ and $b_{i} \in \mathbb{R}$.

Lemma 1: A PWA lifting is continuous. Moreover, it is convex if:

$$
z(x)>a_{j}^{\top} x+b_{j}, x \in \mathbb{X} \backslash X_{j}, \text { holds. }
$$

Since the sets $X_{i} \subset \mathbb{X}$ are compact and cover the space without gaps, there are pairs $(i, j)$ with $X_{i} \cap X_{j} \neq \emptyset$. For each $x \in X_{i} \cap X_{j}$ from the uniqueness of $z(x)$ w.r.t parameters $a_{i}$ and $b_{i}$ it follows $a_{i}^{\top} x+b_{i}=a_{j}^{\top} x+b_{j}$ and thus the lifting $i s$ continuous. A necessary and sufficient condition of convexity is that inequality $\lambda z\left(x_{1}\right)+(1-\lambda) z\left(x_{2}\right) \geq z\left(\lambda x_{1}+(1-\lambda) x_{2}\right)$ holds for any pair $x_{1}, x_{2} \in \mathbb{X}$ and $\lambda \in[0,1]$. Without loss of generality let us assume that $x_{1} \in X_{i}, x_{2} \in X_{j}$ and $\lambda x_{1}+$ $(1-\lambda) x_{2} \in X_{k}$. Thus, the inequality becomes $\lambda\left(a_{i}^{\top} x_{1}+b_{i}\right)+$ $(1-\lambda)\left(a_{j}^{\top} x_{2}+b_{j}\right) \geq a_{k}^{\top}\left(\lambda x_{1}+(1-\lambda) x_{2}\right)+b_{k}$. Rewriting the right-hand side as $\lambda\left(a_{k}^{\top} x_{1}+b_{k}\right)+(1-\lambda)\left(a_{k}^{\top} x_{2}+b_{k}\right)$ and applying (9) shows that the inequality holds, thus (8) is convex.

Theorem 1: A piecewise affine lifting is continuous and convex iff $\left(a_{i}, b_{i}\right)$ satisfy:

$$
\begin{aligned}
& a_{i}^{\top} v+b_{i} \geq a_{j}^{\top} v+b_{j}+\epsilon, \forall v \in \mathcal{V}\left(P_{i}\right), \forall i \neq j, \\
& a_{i}^{\top} v+b_{i} \leq M, \forall v \in \mathcal{V}\left(P_{i}\right), \forall i .
\end{aligned}
$$

In (10) $\epsilon, M>0$ are suitably chosen and $\mathcal{V}\left(P_{i}\right)$ denotes the collection of extreme points of $P_{i}$.

Let us recall that $P_{i} \subset \operatorname{int}\left(X_{i}\right)$ are convex. Thus, with a suitably chosen $\epsilon$, and checking (10b), (10a) guarantees the inequality:

$$
a_{i}^{\top} x+b_{i}>a_{j}^{\top} x+b_{j}, x \in \mathbb{X} \backslash X_{j},
$$

which implies the continuity and convexity (through Lemma 1) of lifting (8), thus concluding the proof.

Taking $P_{i}$ as polyhedral sets (i.e, having a finite number of extreme points), allows to constructively obtain the lifting as the result of the following convex optimization problem:

$$
\min _{a_{i}, b_{i}} \sum_{i=1}^{N_{o}}\left\|\left[\begin{array}{ll}
a_{i} & b_{i}
\end{array}\right]^{\top}\right\|_{2}^{2} \text { s.t. (10a) - (10b) hold. }
$$

Based on the solutions of (12), we define the following "d+1"-dimensional polyhedron:

$$
\mathcal{P}=\left\{\left[\begin{array}{l}
x \\
z
\end{array}\right] \in \mathbb{R}^{d+1}:\left[a_{i}^{\top}-1\right]\left[\begin{array}{l}
x \\
z
\end{array}\right] \leq-b_{i}, i \in \mathcal{I}\right\} .
$$

Projecting the facets of $\mathcal{P}$ on $\mathbb{X}$ provides a polyhedral partition $\left\{X_{i}\right\}_{i=1: N_{o}}$.

Corollary 1: The polyhedral partition $\left\{X_{i}\right\}_{i=1: N_{o}}$ has the following properties:

i) $P_{i} \subset \operatorname{int}\left(X_{i}\right), \forall i$,

ii) $X_{i} \cap P_{j}=\emptyset, \forall j \neq i$.

Projecting the facets ${ }^{3}$ of $\mathcal{P}$ on $\mathbb{X}$, we obtain the polyhedral partition $\left\{X_{i}\right\}_{i=1: N_{o}}$ :

$$
X_{i}=\operatorname{proj}\left(\mathcal{F}_{i}^{d-1}(\mathcal{P}), \mathcal{X}\right), \forall i,
$$

which allows proving the properties: i) The feasibility of (12) and the vertex-representation of $P_{i}, \forall i$, implies that for all $x \in P_{i}$ we have: $\left[\begin{array}{c}x \\ z(x)\end{array}\right]=\left[\begin{array}{c}x \\ a_{i}^{\top} x+b_{i}\end{array}\right] \in \mathcal{P}$. Thus, the lifting corresponding to $P_{i}$ is included in $\mathcal{F}_{i}^{d-1}(\mathcal{P})$. By projecting over $\mathbb{X}$, it directly leads to inclusion $P_{i} \subset X_{i}$.

ii) Let us suppose that there is a $y \in X_{i} \cap P_{j}$. According to i) we have $P_{j} \subset X_{j}$. That leads to $y \in X_{j}$ and, from feasibility of (10a):

$$
a_{j}^{\top} y+b_{j} \geq a_{i}^{\top} y+b_{i}+\epsilon .
$$

Moreover, $y \in X_{i} \cap P_{j}$ means that $y \in X_{i} \cap X_{j}$. Thus, via (Lemma 1), we have:

$$
a_{i}^{\top} y+b_{i}=a_{j}^{\top} y+b_{j}
$$

Combining (14) and (15), gives $\epsilon \leq 0$, which contradicts that $\epsilon>0$.

Remark 2: In contrast with [14], lifting (12) uses the vertices of the obstacle $P_{i}$ instead of vertices of the partition region $X_{i}$.

Remark 3: The feasibility of (12) implies the existence of a convex liftable partition of $\mathbb{X}$. The infeasibility of (12) does not exclude the existence of either a convex non-liftable or non-convex liftable partition [14]. However, the likelihood of such partitions is minor compared to convex liftable ones (see Section $\mathrm{V}$ for further discussions on this topic).

Fig. 2 depicts the partitioning of a 2D multi-obstacle environment and the corresponding lifting. Note that there exist a one-to-one relationship between obstacles and partition cells. Furthermore, in Fig. 3 we delineate the partitioning of a more

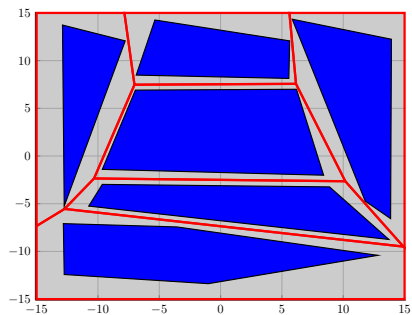

(a) $\left\{X_{i}\right\}_{i=1: N_{o}}$ for $N_{o}=6$

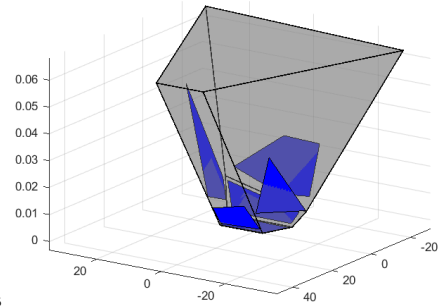

(b) $\mathbb{P}$ and embeddings of the obstacles
Fig. 2: Space-partitioning and the lifting as in Corollary 1.

\footnotetext{
${ }^{3}$ Facet $\mathcal{F}_{i}^{d-1}(\mathcal{P})$ is the $(d-1)$-order face of polyhedron $\mathcal{P}$, i.e., the inequalities describing $\mathcal{P}$ remain the same except the $i$-th which is converted to an equality.
} 
complex 2D environment and a 3D case, as well. For ease of the presentation we restricted the illustrations to $2 \mathrm{D} / 3 \mathrm{D}$, but the constructive result Corollary 1 holds also for arbitrary dimensions. As a side remark, the construction based on lifting is extremely fast (LP) and, thus, can be evaluated for moving obstacles (however, this scenario is outside the scope of the present paper).

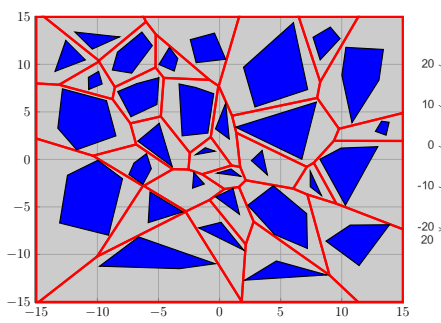

(a) $N_{o}=31, d=2$

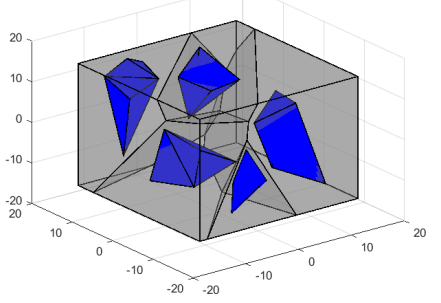

(b) $N_{o}=5, d=3$
Fig. 3: Space-partitioning $\left\{X_{i}\right\}_{i=1: N_{o}}$ from (12).

\section{GEOMETRIC PATH GENERATION}

The partitioning introduced in the previous section induces a graph structure which allows to compute an obstacleavoiding path.

Definition 5: A weighted graph $\Gamma(\mathcal{N}, \mathcal{E}, f)$ is defined by the triple $(\mathcal{N}, \mathcal{E}, f)$ with $\mathcal{N}$ the set of nodes, $\mathcal{E}$ the set of edges, and $f: \mathcal{E} \rightarrow \mathbb{R}$ a function which associates to each edge a real positive value (its weight).

Remark 4: A sometimes encountered variation is for the weight function $f($.$) to associate weights to the nodes ( f$ : $\mathcal{N} \rightarrow \mathbb{R}$ ), not to the edges, as is the case in Def. 5. Consequently, the classical shortest-path algorithms are slightly modified but can still be applied.

Having the partition $\left\{X_{i}\right\}_{i=1: N_{o}}$ of the workspace $\mathbb{X}$, the goal is to construct a graph in order to generate feasible paths through $\mathbb{X}$. Therefore, we have to select the nodes, the edges and the associated weights from the constructive parameters of the compact sets $X_{i}$ (vertices and faces). In Table I we delineate the existing possibilities for selecting graph components. While for the $d=2$ the differences among the alternatives are negligible, for higher dimensions the choice of the graph representation may have a significant effect on the characteristics of the corridor (7).

\begin{tabular}{|c|c|c|c|}
\hline & Domain of $f$ & $\overline{\mathcal{N}}$ & $\mathcal{E}$ \\
\hline 1 & \multirow{2}{*}{$\mathcal{E}$} & $\mathcal{F}^{0}\left(X_{i}\right)$ & $\mathcal{F}^{1}\left(X_{i}\right)$ \\
\hline 2 & & $\mathcal{F}^{d-2}\left(X_{i}\right)$ & $\mathcal{F}^{d-1}\left(X_{i}\right)$ \\
\hline 3 & \multirow{2}{*}{$\mathcal{N}$} & $\mathcal{F}^{1}\left(X_{i}\right)$ & $\mathcal{F}^{0}\left(X_{i}\right)$ \\
\hline 4 & & $\mathcal{F}^{d-1}\left(X_{i}\right)$ & $\mathcal{F}^{d-2}\left(X_{i}\right)$ \\
\hline
\end{tabular}

TABLE I: Alternative selection of graph triple $(\mathcal{N}, \mathcal{E}, f)$.

Proposition 1: $\Gamma\left(\left\{\mathcal{F}^{d-2}\left(X_{i}\right)\right\}_{X_{i} \in \mathbb{X}},\left\{\mathcal{F}^{d-1}\left(X_{i}\right)\right\}_{X_{i} \in \mathbb{X}}, f\right)$ is a connected planar graph.

As $\left\{X_{i}\right\}_{i=1: N_{o}}$ is a partition of $\mathbb{X}$ satisfying ii) from Def. 2, there exists no intersection among the facets of regions $X_{i}$ $\left(\mathcal{F}^{d-1}\left(X_{i}\right)\right)$, except the faces of dimension $d-2$, and, by consequence, among edges of the graph (i.e. $\Gamma$ is planar). Also, ii) from Def. 2 means that for any pair of " $d-2$ "-faces from those of partition $\left\{X_{i}\right\}_{i=1: N_{o}}$ there exists a sequence of facets which connect these two faces. Hence, the existence of a path between any two nodes through the graph is guaranteed (i.e. $\Gamma$ is connected).

For illustration, we present a constructive method for the graph $\Gamma_{1}\left(\mathcal{N}_{1}, \mathcal{E}_{1}, f_{1}\right)$, in the case $d=2$, where:

- the nodes are the vertices of the polyhedral regions $X_{i}$ :

$$
\mathcal{N}_{1}=\bigcup_{i=1}^{N_{o}} \mathcal{F}^{0}\left(X_{i}\right)=\bigcup_{i=1}^{N_{o}} \mathcal{V}\left(X_{i}\right)
$$

- the edges are the facets of the partition regions $X_{i}$ (i.e., their support hyperplanes):

$$
\mathcal{E}_{1}=\mathcal{F}^{1}\left(X_{i}\right)
$$

- the function $f_{1}$ gives the Euclidean distance between the incident nodes of the edge.

We depict in Fig. 4a, the graph corresponding to the polyhedral partition from Fig. $3 \mathrm{a}$ and in Fig. 4b, the graph associated with the $3 \mathrm{D}$ partitioning shown in Fig. $3 \mathrm{~b}$.

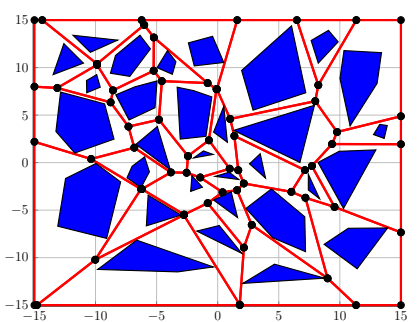

(a) $d=2$

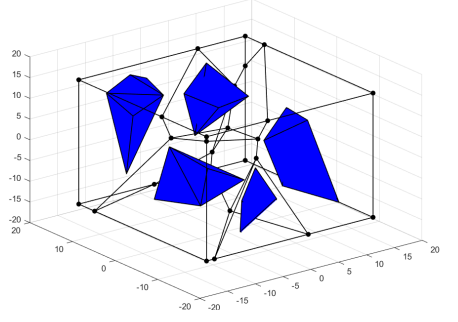

(b) $d=3$
Fig. 4: Graph $\Gamma(\mathcal{N}, \mathcal{E}, f)$.

To determine a corridor as in (7), the graph $\Gamma$ needs to be slightly modified. Specifically, we have to add the nodes/edges corresponding to the start and final points $x_{0}, x_{f} \in \operatorname{int}\left(\mathcal{C}_{\mathbb{X}}(\mathbb{P})\right)$. Usually, this augmentation of the graph involves some auxiliary components as well. For instance, in the $2 \mathrm{D}$ case of $\Gamma_{1}$ a first step consist in finding the closest edge such that the connection to it does not intersect any obstacles. Therefore, we compute the closest projection on the facets $\mathcal{F}^{1}\left(X_{i}\right)$ which satisfies (16b):

$$
\begin{array}{r}
\left(j^{\perp}, x^{\perp}\right)\left(x_{0}\right) \quad=\arg \min _{j \in \mathcal{I}_{f_{i}} \in \operatorname{F}_{j}^{1}\left(X_{i}\right)}\left\|x-x_{i}\right\| \\
\text { s.t. } \quad \alpha x+(1-\alpha) x_{0} \notin P_{i}, \forall \alpha \in[0,1], \\
x_{0} \in X_{i},
\end{array}
$$

where $\mathcal{I}_{f_{i}}=1, \ldots, N_{f_{i}}$ with $N_{f_{i}}$ the number of facets of $X_{i}$. Similarly, we obtain $\left(j^{\perp}, x^{\perp}\right)\left(x_{f}\right)$. These two auxiliary nodes are added to the graph by linking them with the incident nodes of the containing edge and removing this edge. A new graph $\tilde{\Gamma}_{1}\left(x_{0}, x_{f}\right)$ which preserves the properties of $\Gamma_{1}$ is thus obtained. A graph search algorithm (e.g. Dijkstra's Algorithm [9]) is employed and the shortest path between the nodes induced by $x_{0}$ and $x_{f}$ is obtained. 
Remark 5: For further use, we denote the shortest path through the graph between $x_{i}$ and $x_{f}$ as $\operatorname{Path}\left(x_{0}, x_{f}\right)=$ $\left(\bar{x}_{0}=x_{i}, \bar{x}_{1}, \ldots, \bar{x}_{n}, \bar{x}_{n+1}=x_{f}\right)$. This represents an ordered set of points where no segment defined by a pair of consecutive points cuts any of the obstacles. It is not a path in the sense stated in problem P3), but is a sufficient condition for the existence of a corridor (7).

Proposition 2: Any polyhedral partition $\left\{X_{i}\right\}_{i=1: N_{o}}$ provides a corridor (7) for a given pair $x_{0}, x_{f} \in \operatorname{int}\left(\mathcal{C}_{\mathbb{X}}(\mathbb{P})\right)$. As Proposition 1 states, there exists a connected graph $\Gamma$ induced by the partition $\left\{X_{i}\right\}_{i=1: N_{o}}$. By connecting any two points to the graph the connectivity is preserved. Thus, by using a search algorithm for the augmented graph we identify a continuous piecewise affine function $\gamma$ generated by the edges composing $\operatorname{Path}\left(x_{0}, x_{f}\right)$. Moreover, we consider $\rho$ as a PW constant function defined for each edge of $\operatorname{Path}\left(x_{0}, x_{f}\right)$ as the minimum Hausdorff distance between the edge and the obstacles around it. The set $\Pi$, described by functions $\gamma$ and $\rho$ is a corridor in the sense of (7).

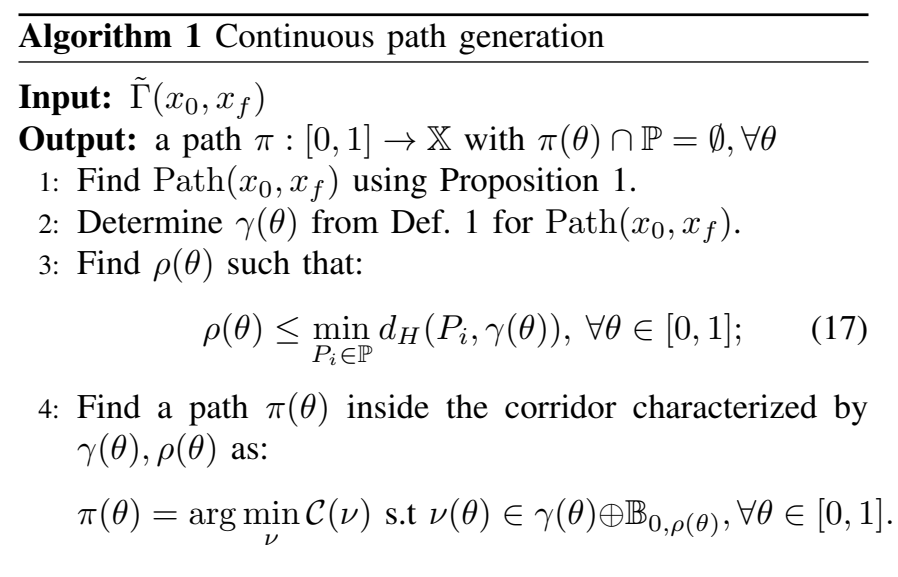

Each step of Algorithm 1 represents a further refinement along the trajectory generation procedure. Step 1 provides the graph structure of the workspace, from which, in Step 2, $\gamma(\theta)$, a PWA (continuous) function is given (under the construction proposed in this section: a collection of edges). To these, in Step 3 is attached, $\rho(\theta)$, a continuous width function which provides a measure of the acceptable deviation from the nominal $\gamma(\theta)$. Step 4 replaces the feasible solution $\gamma(\theta)$ by an optimization-based selection $\pi(\theta)$. The cost $\mathcal{C}(\nu)$ is often taken as the path length but may be replaced/augmented by a cost describing energy/performance/smoothness.

Remark 6: Solving a continuous optimization problem, as is required in Steps 3 and 4, is often difficult if not downright infeasible. Standard approaches are to sample the continuous variables ( $\theta$ in our case) or to project along a basis function and reformulate the problem in terms of the associated weights.

For illustration purposes we revisit the obstacle collection shown in Fig. 3a to which we apply Algorithm 1.

First, we construct the associated graph (depicted in Fig. 4a) and find a path $\gamma$, as shown in Fig. 5. Next, we provide an approximation of the corridor width $\rho$ (gray area in Fig. 5 is the corridor, as defined in (7)). To compute the

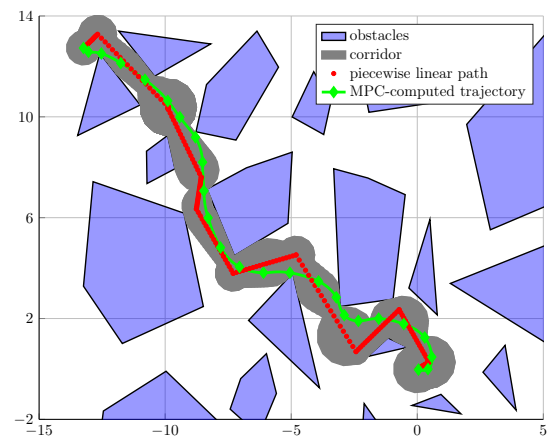

Fig. 5: The shortest path $\operatorname{Path}\left(x_{i}, x_{f}\right)$ and a feasible trajectory within the corridor.

corridor width we sampled the continuous parameter $\theta$ and introduced it in (17). We sidestep Step 4 of the algorithm by choosing $\pi=\gamma$. This path is provided as reference to a standard path tracking mechanism which (green line with diamond markers) is shown to respect the constraints (there is no intersection with the obstacles and the destination is successfully reached). For illustration of the ultimate path tracking task we considered a standard double integrator dynamic and applied an MPC (Model Predictive Control) strategy.

\section{BEYOND THE CONVEX LIFTABLE CASE}

The previous sections assume the feasibility of (12) based on the initial geometry of the obstacles. There exist however obstacles which either are enclosed by not liftable partition or are non-convex themselves, and thus do not fulfill the assumptions (see Fig. 6).

For the convex-non-liftable cluttered environments, the solution comes from the structural result presented in [19] which can be summarized as follows: for any polyhedral partition there always exists one subdivision such that the internal boundaries of this partition are preserved and the new partition is convexly liftable. Transposed in the present framework of obstacles included in the partition's regions, it follows that obstacles can be subdivided as collections of convex subsets, thus enabling convex lifting.

Remark 7: Feasibility of the convex lifting comes at the price of additional edges in the planar graph studied in Proposition 1. However, the removal of these edges at a postprocessing phase is trivial as long as they intersect at least one of the original obstacles.

The objective in the case of non-convex obstacles is to express these regions in terms of finite unions of convex obstacles. Practically, this leads to a similar procedure as for the non-liftable case. However, the lack of convexity in the original setting can lead to structural infeasibility as illustrated on the configuration depicted in Fig. $6 \mathrm{~b}$ and resumed next.

Remark 8: The replacement of non-convex obstacles by a union of convex obstacles enables the construction of a partition as in Section III. However, the removal of edges which intersect the original obstacles can lead to a disconnected 
graph. The loss of connectivity in the graph shows that there exist pairs of points between which there is no feasible path (at least through the methods discussed here).

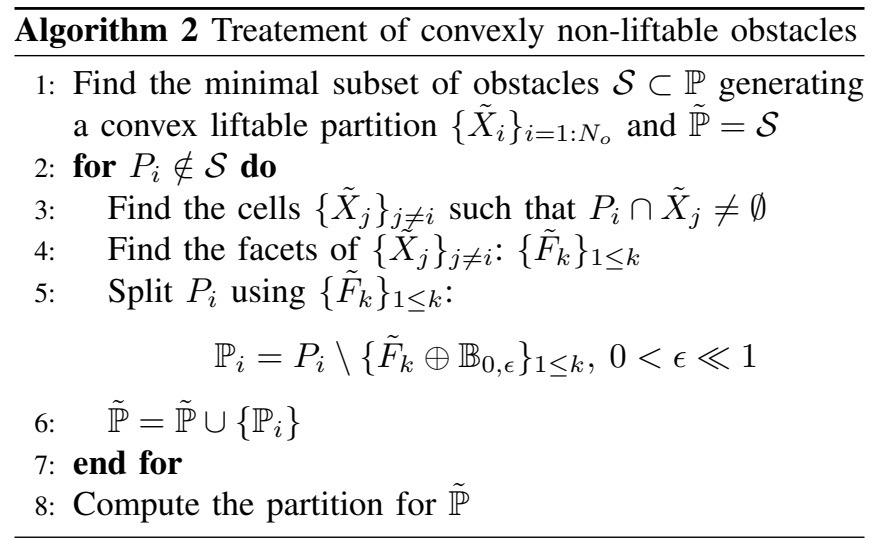

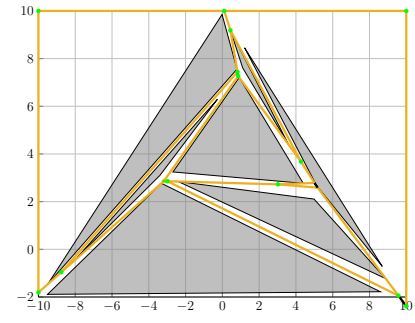

(a) convex non-liftable

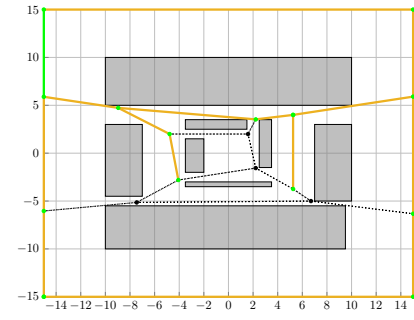

(b) non-convex obstacles
Fig. 6: Convex liftable representation of originally nonliftable obstacles and their associated partition.

\section{CONCLUSIONS}

The paper presents a constructive solution for the generation of a path between two points in an environment obstructed by multiple obstacles in a $d$-dimensional space. The global information on the geometry of the obstacles is considered as an entry point for a convex optimization procedure which leads to a convex lifting allowing the partitioning of the cluttered environment. This partitioning is a key element for describing a graph around the obstacles and ultimately for the generation of corridors which avoid obstacles. From the computational point of view, the validity of the construction relies on the feasibility of the convex lifting procedure. It was shown that feasibility can be improved by a reformulation of the obstacles in terms of a finite number of convex subsets. Furthermore, this principle allows the generalization of the construction for path planning in the presence of multiple non-convex obstacles. In the latter case, a disconnected graph within the generation of the corridors will certify the infeasibility of the of the path planning problem for at least a pair of points in the cluttered environment.

\section{ACKNOWLEDGMENT}

The research of Daniel Ioan is financially supported by the Ministry of the Armed Forces - Defence Procurement
Agency (DGA) - no.2017352. The authors acknowledge Jacques Blanc-Talon, expert DGA, for the fruitful discussions.

The research of Florin Stoican is supported by the Politehnica University of Bucharest, through the internal research grant GNaC 2918 ARUT, contract no. 4/15.10.2018.

The authors would like to thank the reviewers for all of their valuable and insightful comments.

\section{REFERENCES}

[1] F. Janecek, M. Klauco, M. Kaluz, and M. Kvasnica, "OPTIPLAN: A Matlab Toolbox for Model Predictive Control with Obstacle Avoidance," IFAC-PapersOnLine, vol. 50, no. 1, pp. 531-536, Jul. 2017.

[2] A. Puri, "A survey of unmanned aerial vehicles (uav) for traffic surveillance," Department of computer science and engineering, University of South Florida, pp. 1-29, 2005.

[3] A. Richards and J. P. How, "Aircraft trajectory planning with collision avoidance using mixed integer linear programming," in Proceedings of the 2002 American Control Conference., vol. 3. IEEE, 2002, pp. 1936-1941.

[4] I. Prodan, F. Stoican, S. Olaru, and S.-I. Niculescu, "Mixed-integer representations in control design: Mathematical foundations and applications." Springer, 2015.

[5] Y.-b. Chen, G.-c. Luo, Y.-s. Mei, J.-q. Yu, and X.-1. Su, "UAV path planning using artificial potential field method updated by optimal control theory," International Journal of Systems Science, vol. 47, no. 6, pp. 1407-1420, Apr. 2016.

[6] R. Olfati-Saber and R. M. Murray, "Distributed cooperative control of multiple vehicle formations using structural potential functions," in IFAC world congress, vol. 15, no. 1, 2002, pp. 242-248.

[7] E. Rimon and D. E. Koditschek, "Exact robot navigation using artificial potential functions," Departmental Papers(ESE), p. 323, 1992.

[8] M. Szmuk, C. A. Pascucci, D. Dueri, and B. Acikmese, "Convexification and real-time on-board optimization for agile quad-rotor maneuvering and obstacle avoidance," in 2017 IEEE/RSJ International Conference on Intelligent Robots and Systems (IROS), sep 2017.

[9] S. Karaman and E. Frazzoli, "Sampling-based algorithms for optimal motion planning," The International Journal of Robotics Research, vol. 30, no. 7, pp. 846-894, Jun. 2011.

[10] S. M. LaValle, "Rapidly-exploring random trees: A new tool for path planning," Tech. Rep., 1998.

[11] G. Franzè and W. Lucia, "The obstacle avoidance motion planning problem for autonomous vehicles: A low-demanding receding horizon control scheme," Systems \& Control Letters, vol. 77, pp. 1-10, 2015.

[12] R. J. M. Afonso, R. K. H. Galvao, and K. H. Kienitz, "Waypoint trajectory planning in the presence of obstacles with a tunnel-milp approach," in 2013 ECC. IEEE, 2013, pp. 1390-1397.

[13] S. Liu, M. Watterson, K. Mohta, K. Sun, S. Bhattacharya, C. J. Taylor, and V. Kumar, "Planning dynamically feasible trajectories for quadrotors using safe flight corridors in 3-d complex environments," IEEE Robotics and Automation Letters, vol. 2, no. 3, pp. 1688-1695, jul 2017. [Online]. Available: https://doi.org/10.1109/lra.2017.2663526

[14] N. A. Nguyen, M. Gulan, S. Olaru, and P. Rodriguez-Ayerbe, "Convex lifting: Theory and control applications," IEEE Transactions on Automatic Control, vol. 63, no. 5, pp. 1243-1258, 2018.

[15] T. Faulwasser, B. Kern, and R. Findeisen, "Model predictive pathfollowing for constrained nonlinear systems," in Proceedings of the 48h IEEE Conference on Decision and Control (CDC) held jointly with 2009 28th Chinese Control Conf. IEEE, 2009, pp. 8642-8647.

[16] S. Boyd and L. Vandenberghe, Convex optimization. Cambridge university press, 2004.

[17] X. Wang, M. Kloetzer, C. Mahulea, and M. Silva, "Collision avoidance of mobile robots by using initial time delays," in 2015 54th IEEE Conference on Decision and Control (CDC), Dec. 2015, pp. 324-329.

[18] K. Sugihara, "Approximation of generalized voronoi diagrams by ordinary voronoi diagrams," CVGIP: Graphical Models and Image Processing, vol. 55, no. 6, pp. 522-531, 1993.

[19] N. A. Nguyen, S. Olaru, P. Rodriguez-Ayerbe, M. Hovd, and I. Necoara, "Fully inverse parametric linear/quadratic programming problems via convex liftings," in Developments in Model-Based Optimization and Control. Springer, 2015, pp. 27-47. 